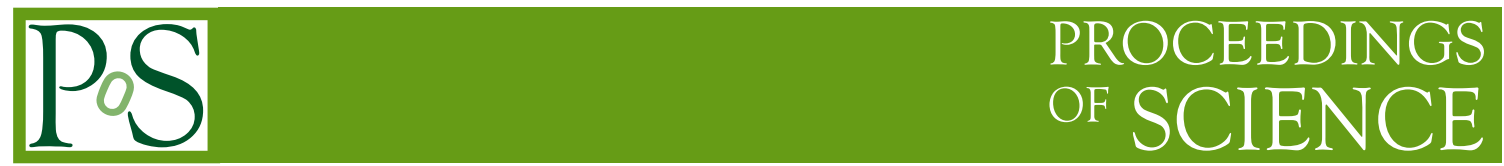

\title{
Deep exclusive reactions at Jefferson Lab Hall A
}

\author{
Carlos Munoz Camacho* \\ LPC-Clermont, CNRS/IN2P3 \\ E-mail: munoz@jlab.org
}

Recent results on the Generalized Parton Distribution (GPD) program at Jefferson Lab (JLab) will be presented. The emphasis will be in the Hall A program aiming at measuring $Q^{2}-$ dependences of different terms of the Deeply Virtual Compton Scattering (DVCS) cross section. This is a fundamental step before one can extract GPD information from JLab DVCS data. The upcoming program in Hall A, using both a $6 \mathrm{GeV}$ beam $(2010)$ and a $11 \mathrm{GeV}$ beam $(\approx 2015)$ will also be described.

XVIII International Workshop on Deep-Inelastic Scattering and Related Subjects April 19 -23, 2010

Convitto della Calza, Firenze, Italy

*Speaker. 


\section{Introduction}

Understanding of the fundamental structure of matter requires an understanding of how quarks and gluons are assembled to form the hadrons.

The asymptotic freedom of Quantum Chromodynamics (QCD) makes it possible to use perturbation theory to treat interactions of quarks and gluons at short distances. In high-energy scattering, short-distance and long-distance physics may be separated to leading power in momentum transfer-an approach known as "factorization". For example, the cross sections for hard electronproton or proton-proton collisions that transfer large momentum can be expressed as a product of a short-distance partonic (quark or gluon) cross section, calculable in perturbative $\mathrm{QCD}$, and parton distribution functions that encode the long-distance information on the structure of the proton.

Traditionally, the spatial view of nucleon structure provided by lattice QCD and the momentumbased view provided by the parton picture stood in stark contrast. Recent theoretical developments have clarified the connection between these two views, that of momentum and that of spatial coordinates, such that ultimately it should be possible to provide a complete "space-momentum" map of the proton's internal landscape. These maps, referred to as Generalized Parton Distributions (GPDs), describe how the spatial shape of a nucleon changes when probing different ranges of quark momentum. Projected along one dimension, GPDs reproduce the form factors; along another dimension they provide a momentum distribution. With enough information about the correlation between space and momentum distributions, one can construct a full "tomographic" image of the proton. The weighted integrals, or moments, of the GPDs contain information about the forces acting on the quarks bound inside the nucleon. These moments can now be computed using lattice QCD methods. Not only do they have an attractive physical interpretation, but they can also be compared directly to unambiguous predictions from the underlying theory. Higher-order QCD corrections for many observables sensitive to GPDs have now also been calculated, strengthening the theoretical underpinning for extraction of GPDs.

The simplest and cleanest experimental process that gives access to GPDs is Deeply Virtual Compton Scattering (or DVCS), where a virtual photon of high 4-momentum $Q^{2}$ scatters off a nucleon producing a real photon on the final state $\left(\gamma^{*} p \rightarrow \gamma p\right)$. Experimentally, DVCS occurs in the electroproduction of photons off the nucleon: $e p \rightarrow e p \gamma$, where there is a competing channel, the Bethe-Heitler $(\mathrm{BH})$ process, where the photon of the final state is radiated by the electron and not the proton. The BH process is, however, calculable in Quantum Electrodynamics given our knowledge of the proton form factors.

Extracting GPDs from DVCS requires the fundamental demonstration that DVCS is well described by the leading twist mechanism at finite $Q^{2}$. This leading twist mechanism consists on the scattering on a single quark (or gluon) inside the nucleon.

\section{Current experimental situation}

The H1 [1, 2] and ZEUS [3] collaborations measured the cross section for $x_{B} \approx 10^{-3}$. The HERMES collaboration measured relative beam-helicity [4] and beam-charge asymmetries [5, 6]. Relative beam-helicity [7] and longitudinal target [8] asymmetries were measured at the Thomas Jefferson National Accelerator Facility (JLab) by the CLAS collaboration. The first dedicated 
DVCS experiment ran in Hall A at JLab [9]. Helicity-correlated cross section of electroproduction of photons were measured with high statistical accuracy as a function of $Q^{2}$ (Fig. 1).

The combination of Compton form factors (CFF) extracted from these data (Fig. 2) was found to be independent of $Q^{2}$, within uncertainties. This exciting result strongly supports the twist-2 dominance of the imaginary part of the DVCS amplitude. GPDs can then be accessed experimentally at the kinematics of JLab.

A dedicated DVCS experiment on the neutron (E03-106) followed E00-110. Using a deuterium target, E03-106 provided the first measurements of DVCS on the neutron, particularly sensitive to the least known GPD $E$, and DVCS on the deuteron [10]. A first constraint on the orbital angular momentum of quarks inside the nucleon (Fig. 3) was obtained relying on the VGG model of GPDs [11].

\section{Future DVCS program with CEBAF at $6 \mathrm{GeV}$}

A new experiment E07-007 was recently approved with the highest scientific rating (A) by the JLab Program Advisory Committee (PAC-31) and will run later this year. E07-007 will use the important lessons we learned in E00-110 to further test and explore the potential of DVCS to measure GPDs.

In E00-110 we managed to determine the photon electroproduction helicity-independent (unpolarized) cross section. This additional information was not in the initial goal of the E00-110 experiment, and we were only able to measure it at one single value of $Q^{2}=2.3 \mathrm{GeV}^{2}$ (Fig. 1). We found that the total cross section was much larger than the BH contribution. The excess in the cross section is then coming from both the interference term (BH-DVCS) and the DVCS ${ }^{2}$ contribution. These contributions contain different kinds of GPD combinations. The interference term is proportional to a linear combination of GPD integrals, whereas the $\mathrm{DVCS}^{2}$ is related to a bilinear combination of GPD integrals.

The harmonic $\phi$ structure of the DVCS cross section does not allow the independent determination of the BH-DVCS interference and the $\mathrm{DVCS}^{2}$ contributions.

\subsection{Interference and DVCS ${ }^{2}$ separation}

E07-007 was approved to measure the photon electroproduction helicity-independent cross section as a function of $Q^{2}$ and at different incident beam energies. The different beam energy dependence of the DVCS ${ }^{2}$ and BH.DVCS interference term complement the azimuthal analysis of the cross section in order to provide a complete separation of these two contribution.

\subsection{Separation of the longitudinal $\pi^{0}$ electroproduction cross section}

In addition to these fundamental measurements on DVCS, E07-007 will concurrently measure the $\pi^{0}$ electroproduction cross section for different beam energies. We successfully measured the $\pi^{0}$ electroproduction cross section in E00-110. However, since we only ran at one incident beam energy, the longitudinal and transverse separation of the cross section could not be done. E07-007 will allow the first separation of the longitudinal cross section, using the Rosenbluth technique. A factorization theorem holds for the longitudinal $\pi^{0}$ electroproduction cross section that relates it, at sufficiently large $Q^{2}$, to a different flavor combination of GPDs. This channel is an essential 

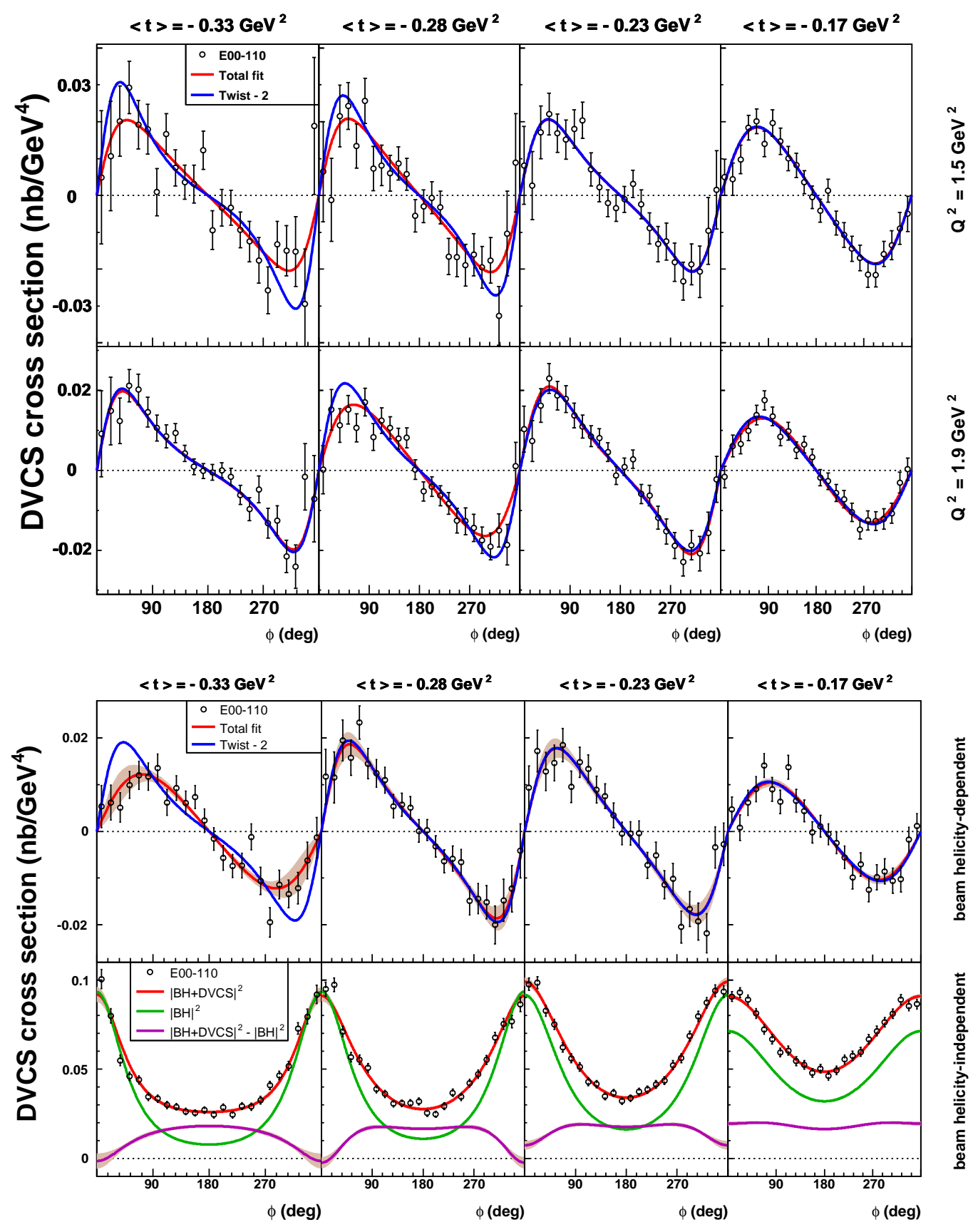

Figure 1: Data and fit to the helicity-dependent $d^{4} \Sigma / d Q^{2} d x_{B} d t d \phi$, and the helicity-independent $d^{4} \sigma /\left[d Q^{2} d x_{B} d t d \phi\right]$ cross sections, as a function of the azimuthal angle $\phi$ between the leptonic and hadronic planes. All bins are at $\left\langle x_{B}\right\rangle=0.36$. Error bars show statistical uncertainties. Solid lines show total fits with one- $\sigma$ statistical error bands. The green line is the $|\mathrm{BH}|^{2}$ contribution to $d^{4} \sigma$. The first three rows show the helicity-dependent cross section for values of $Q^{2}=1.5,1.9$ and $2.3 \mathrm{GeV}^{2}$, respectively. The last row shows the helicity-independent cross section for $Q^{2}=2.3 \mathrm{GeV}^{2}$. 


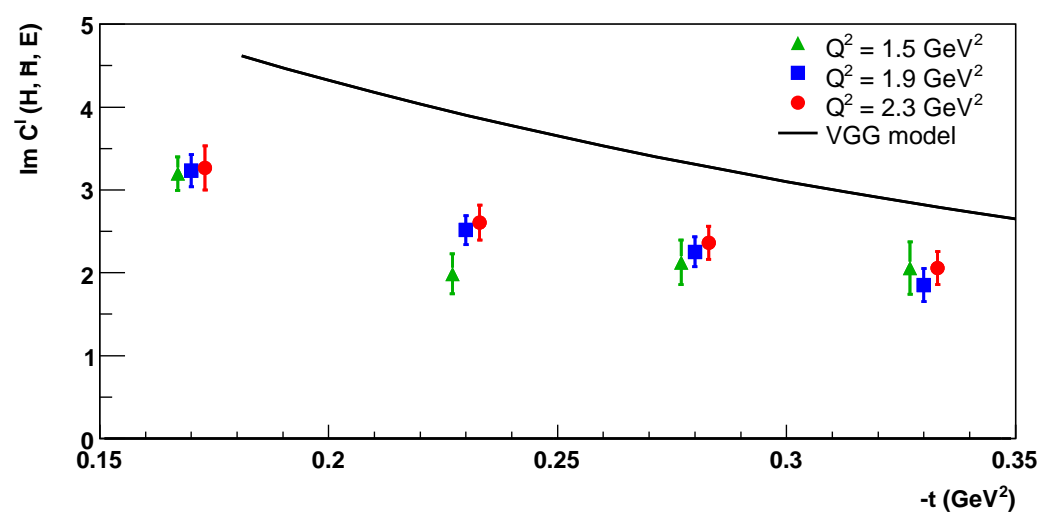

Figure 2: Extracted imaginary part of the twist-2 angular harmonic as function of $t$. Superposed points are offset for visual clarity. Their error bars show statistical uncertainties.

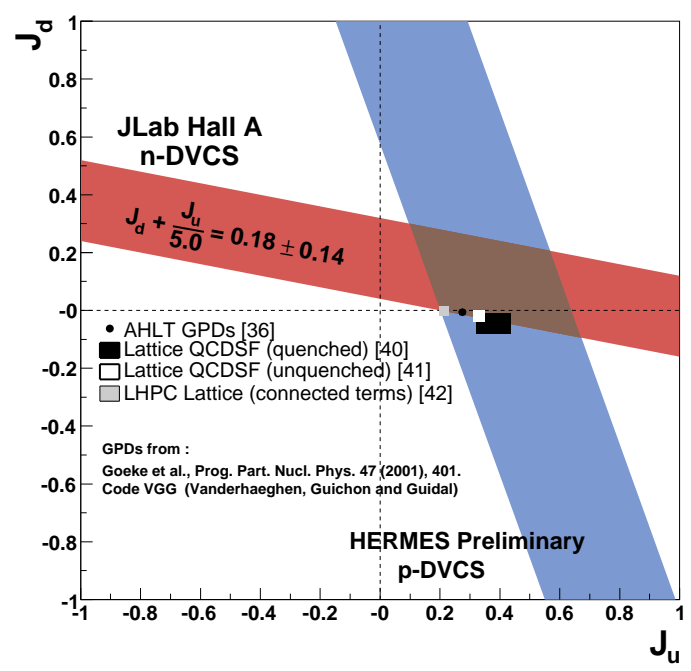

Figure 3: Experimental constraint on $J_{u}$ and $J_{d}$ quark angular momenta from the present n-DVCS results [10]. A similar constraint from the p-DVCS target spin asymmetry measured by HERMES, and different lattice QCD based calculations are also shown.

element to make a flavor decomposition of GPDs, which is impossible to obtain from DVCS on the proton alone.

E07-007 will test the $Q^{2}$-dependence of the longitudinal cross section and check if the factorization is applicable at the $Q^{2}$ values currently accessible experimentally (around $Q^{2} \sim 2 \mathrm{GeV}^{2}$ ). If this test turns out positive, very interesting and complementary information on GPDs will be available from this channel.

\subsection{E08-025: Rosenbluth-like separation on the neutron}

An equivalent DVCS program at $6 \mathrm{GeV}$ using a deuterium target was approved earlier this year and will run concurrently with E07-007. It will allow to separate the interference and DVCS ${ }^{2}$ terms on neutron observables. This will complement E07-007 on the proton. 


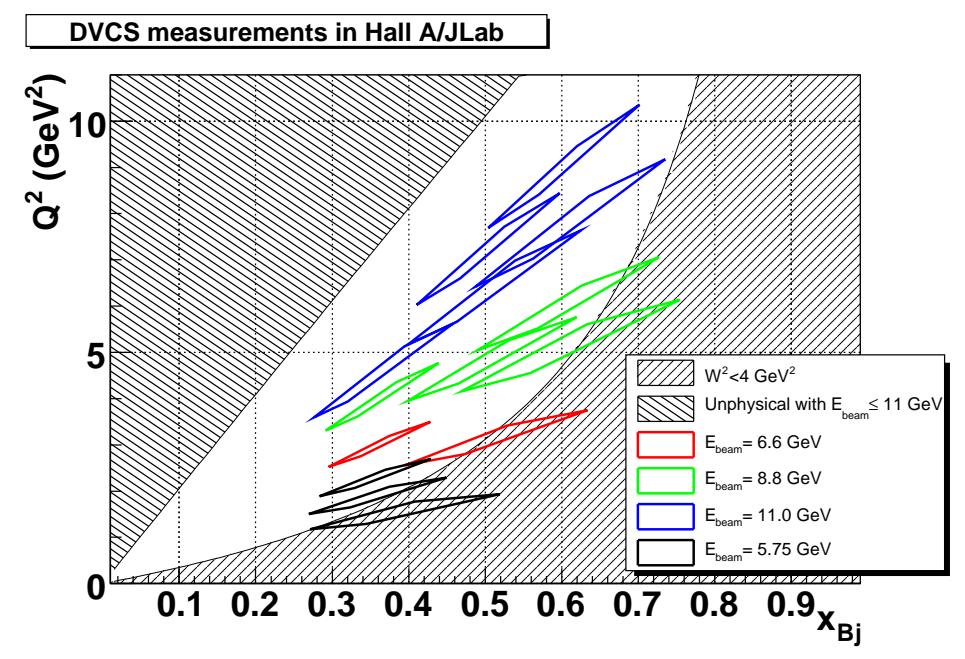

Figure 4: Approved DVCS kinematics for $\mathrm{H}\left(e, e^{\prime} \gamma\right) p$ measurements in Hall A with 3, 4, and 5 pass beams of CEBAF at $12 \mathrm{GeV}$. The diamond shapes trace the approximate acceptance of the Hall A High Resolution Spectrometer in each setting. The boundary of the unphysical region corresponds to the maximum possible $Q^{2}$ at a given $x_{B}$ for $11 \mathrm{GeV}$. This corresponds to $180^{\circ}$ electron scattering, equivalent to $\theta_{q}=0^{\circ}$. The points at $E_{\text {Beam }}=5.75 \mathrm{GeV}$ were obtained in E00-110.

\section{4. $12 \mathrm{GeV}$ program}

The experimental GPD program is at the heart of the scientific motivation of the major Jefferson Lab upgrade to $12 \mathrm{GeV}$. E12-06-114 [12] is a wide experimental program of DVCS high precision cross-section measurements in Hall A with CEBAF at $12 \mathrm{GeV}$, enthusiastically approved by JLab PAC-30. With polarized 6.6, 8.8, and $11 \mathrm{GeV}$ beams incident on the liquid hydrogen target, we will provide a major survey of proton DVCS in almost the entire kinematic range accessible with CEBAF at $12 \mathrm{GeV}$ (Fig. 4).

\section{References}

[1] A. Aktas et al., Eur. Phys. J. C44, 1 (2005).

[2] C. Adloff et al., Phys. Lett. B 517, 47 (2001).

[3] S. Chekanov et al., Phys. Lett. B573, 46 (2003).

[4] A. Airapetian et al., Phys. Rev. Lett. 87, 182001 (2001).

[5] F. Ellinghaus, Nucl. Phys. A711, 171 (2002).

[6] A. Airapetian et al., (2006).

[7] S. Stepanyan et al., Phys. Rev. Lett. 87, 182002 (2001).

[8] S. Chen et al., Phys. Rev. Lett. 97, 072002 (2006).

[9] C. Muñoz Camacho et al., Phys. Rev. Lett. 97, 262002 (2006).

[10] M. Mazouz et al., Phys. Rev. Lett. 99, 242501 (2007).

[11] M. Vanderhaeghen, P. A. M. Guichon, and M. Guidal, Phys. Rev. D60, 094017 (1999).

[12] J. Roche et al., JLab proposal PR12-06-114 (2006). 